\title{
Production of Hybrids Between Arachis hypogaea and A. chiquitana (section Procumbentes) ${ }^{1}$
}

\author{
Nalini Mallikarjuna ${ }^{2}$
}

\begin{abstract}
There is no report of successful crosses between cultivated peanut (Arachis hypogaea L.) and wild species from section Procumbentes. Interspecific hybrids between $A$. hypogaea (section Arachis) and A. chiquitana Krapov., W.C. Gregory \& C.E. Simpson (section Procumbentes) were produced by the application of growth regulators to pollinated pistils, and hybrid plants were obtained for the first time by germinating hybrid embryos in vitro. Eleven hybrids were produced, of which one hybrid did not survive to the flowering stage, but the other hybrids were fertile. The hybridity of the $\mathrm{F}_{1}$ plants was confirmed by SSR analysis and one $\mathrm{BC}_{1}$ plant gave rise to mature seeds. In addition, $A$. chiquitana has been identified as one of the few wild species of Arachis showing resistance to Aspergillus flavus colonization. Aspergillus flavus produces aflatoxin, a carcinogenic agent causing liver cancer, a serious post harvest constraint to peanut production world wide. Initial seed screening of $A$. chiquitana for $A$. flavus showed promise of obtaining hybrids resistant to $A$. flavus colonization, but it is unknown if the interspecific hybrids would also have aflatoxin resistance. This is the first report of obtaining hybrids between $A$. hypogaea and $A$. chiquitana.
\end{abstract}

Key Words: Peanut, groundnut, wild species, interspecific hybridization, embryo germination, Aspergillus flavus, aflatoxin.

According to the published literature on the crossability of the species in the genus Arachis, none of the species outside section Arachis will hybridize with the cultivated peanut (Arachis hypogaea L.) without the aid of in vitro methodology. In many instances when hybrids are produced between $A$. hypogaea and wild species outside the section Arachis, hybrids are completely sterile or, in other words, genetic dead-ends (Stalker and

\footnotetext{
${ }^{1}$ I thank Dr. R. Thakur and his group for screening the material for Aspergillus flavus colonization. I also acknowledge the technical assistance provided by $\mathrm{Mr}$. Balakrishna and Mr. Satyanarayana in the crossing program.

${ }^{2}$ International Crops Research Institute for Semi Arid Tropics (ICRISAT) Patancheru P.O. 502 324, Andhra Pradesh, India, (email: N.Mallikarjuna@CGIAR.ORG).
}

Simpson, 1995). With the development of embryo rescue and tissue culture techniques, hybrids between $A$. hypogaea and A. glabrata Benth., (section Rhizomatosae) have been reported (Mallikarjuna and Sastri 1985, 2002; Shen et al., 1995) Arachis chiquitana Krapov., W.C Gregory and C.E. Simpson, is a wild species collected from Chiquitos province of Santa Cruz in Bolivia, and although it was previously placed in section Erectoides by Gregory and Gregory (1979), it is currently placed in section Procumbentes (Krapovickas and Gregory, 1994).

Aflatoxin contamination of peanut, caused by the Aspergillus flavus group of fungi, is one of the most important constraints to quality peanut production in the semi-arid rainfed areas of the world. This is particularly significant in relation to public health and international trade (Waliyar et al., 1994). In addition to peanuts, aflatoxins are found in many agricultural crops such as corn, cotton, wheat, and rice and consumption of the toxin by humans can lead to liver cancer. Househam and Hunt (1991) also reported a striking association between exposure to aflatoxin in children and both stunting and low weight gain. In West Africa, people are chronically exposed to high levels of aflatoxin starting in the utero and continuing throughout life (Gong et al., 2002). Genetic resistance should be one of the major components in a strategy to manage aflatoxin contamination in peanut, but high levels of genetic resistance to aflatoxin have not been identified in cultivated peanuts. Ghewande et al., (1989) reported accessions of $A$. duranensis Krapov. and W.C. Gregory and A. cardenasii Krapov. And W.C. Gregory as being highly resistant to in vitro seed colonization to Aspergillus flavus. Xue et al., (2004) confirmed resistance in three A. duranensis and two $A$. cardenasii accessions after testing 36 accessions of the two species. Arachis chiquitana is one of the three wild species identified as resistant to Aspergillus flavus Link ex. Fries colonization and subsequent aflatoxin production (Thakur et al, 2000). Pande and Rao (2001) also identified $A$. chiquitana as one of the wild species resistant to late leaf spot (LLS), a fungal disease caused by Phaeoisariopsis personata (Berk. \& M.A. Curtis) Deighton. Hence, developing strategies to cross $A$. hypogaea with $A$. chiquitana not only broadens the genetic base of cultivated peanut 
but will also potentially bring in other desirable genes.

\section{Materials and Methods}

Seeds of $A$. chiquitana with $2 n=2 x=20$, (ICG 11560; collector number 36025; PI 476004; Fig. 1A) from section Procumbentes were obtained from the genetic resources division of ICRISAT and grown in a glasshouse. Seeds of $A$. hypogaea cv. ICGS 44 $(2 n=4 x=40)$ were also grown and maintained in the glasshouse. Cross pollinations using A. hypogaea as the female parent and $A$. chiquitana as the pollen donor were carried out before 10:00 a.m. Emasculations of anthers from flowers were carried out the previous evening. Application of $75 \mathrm{mg} / \mathrm{L}$ of gibberellic acid (GA) at the base of pollinated pistils was mandatory to obtain pods from cross pollinations. A total of 493 pollinations were carried out and 167 pods were harvested between 25 and 30 days after pollination. Pods were then surface sterilized and ovules were extracted under sterile conditions. Ovules which were more than 4.0 to $5.0 \mathrm{~mm}$ long were dissected and the embryos (immature seeds) were cultured directly on the semisolid growth medium (Fig. 1E). The growth medium consisted of MS (Murashige and Skoog, 1962) basal medium with 3\% sucrose plus napthalene acetic acid (NAA; $0.1 \mathrm{mg} / \mathrm{L}$ ) and benzylamino purine (BAP; $1.0 \mathrm{mg} / \mathrm{L}$ ). Ovules less than $4.0 \mathrm{~mm}$ in size were not used in the experiment.

Embryos germinated and gave rise to seedlings (Fig. 1F) with individual or multiple shoots. Shoots were rooted in vitro on rooting medium, consisting of $1 / 2$ MS basal salts (plus sucrose), NAA $(2.0 \mathrm{mg} / \mathrm{L})$, indoleacetic acid (IAA; $1.0 \mathrm{mg} / \mathrm{L}$ ). After $15 \mathrm{~d}$ on rooting medium, shoots were transferred to $1 / 2$ MS basal medium (without growth regulators). Healthy roots developed within $3 \mathrm{wk}$ of culture. Shoots with well-developed roots were transferred to sand and acclimatized under controlled conditions at $24 \mathrm{C}$ and $\mathrm{RH}$ of $72-75 \%$. A month of acclimatization was sufficient to transfer the plants to a glasshouse.

DNA was extracted from young, folded leaflets with Qiagen miniprep kits (Qiagen, Valencia, CA) and amplified by means of 30 pmol primer, $5 \mathrm{ng}$ template DNA, $4 \mathrm{mM} \mathrm{MgCL}_{2}, 0.2 \mathrm{mM}$ dNTPs, $1 \mathrm{U}$ Taq polymerase with $1 \times$ reaction buffer in a total reaction volume of $20 \mu \mathrm{L}$. Reaction conditions were $94 \mathrm{C}$ for $2 \mathrm{~min}, 35$ cycles of $94 \mathrm{C}$ for $45 \mathrm{sec}$, empirically defined annealing temperature of $60 \mathrm{C}$ for $1 \mathrm{~min}, 72 \mathrm{C}$ for $90 \mathrm{sec}$, then a final extension of $10 \mathrm{~min}$ at $72 \mathrm{C}$. Amplification products were visualized on non-denaturing 9\% 29:1 (w/w) polyacrylamide gel followed by silver staining. Silver staining consisted of $3 \mathrm{~min}$ in $\mathrm{H}_{2} \mathrm{O}$, $20 \mathrm{~min}$ in $0.1 \%$ (w/v) CTAB, $15 \mathrm{~min}$ in $0.3 \%$ ammonium solution, $15 \mathrm{~min}$ in a solution of $1 \mathrm{M}$ $\mathrm{NaOH}, 0.1 \%$ silver nitrate and a few drops of $25 \%$ ammonium solution, and a rinse in $\mathrm{H}_{2} \mathrm{O}$, and followed by development in a $1.5 \% \mathrm{NaC}$ solution with $0.02 \%$ by volume formaldehyde solution. The SSR procedure and primers are described in detail in Ferguson et al. (2004). Amplification products were noted as present or absent. The four SSR primers used to distinguish the parents and the hybrids, were as follows:

1. pPGPseq3F1 Forward primer-AGCGATCAATCGGTTTCAAG, fragment length-290 bp. Reverse primer-GAAACGAAACGAAGACCGAA.

2. pPGPseq4D4 Forward primer-CGGCTGTTAGGTAATCAGTTCA, fragment length-187 bp. Reverse primer-TCAACAGGAATAGCTGCACG.

3. pPGPseq2A6 Forward primer-GCTTCTTCGTTGTTGCCTTC, fragment length-249 bp. Reverse primer-TGCCAGTTGTTCATAGCTTCA.

4. pPGPseq4H11 Forward primer-ATCACCATCAGAACGATCCC, fragment length-269 bp. Reverse primer-TTGTAGCCTTCTGGCGAGT.

The ploidy of the derivatives was determined by pollen diameter analysis. Three classes of pollen diameter were observed. Diploids had a diameter of 25 to $27 \mu \mathrm{M}$, triploids were 25 to $29 \mu \mathrm{M}$, and tetraploids had a diameter of 45 to $47 \mu \mathrm{M}$ (Singsit and Ozias-Akins, 1992). Pollen diameter of $A$. hypogaea was between 45 to $47 \mu \mathrm{M}$ and that of $A$. chiquitana was between 25 to $29 \mu \mathrm{M}$. Pollen in triploids which had undergone $2 n$ restitution had 43 to $45 \mu \mathrm{M}$ (and apparently fertile) was comparable to pollen grains of tetraploid plants.

Nineteen seeds were surface sterilized and were uniformly wounded by pricking with a sterile needle to allow the invasion of $A$. flavus spores. Seeds were spray inoculated with $A$. flavus spore suspension $\left(1 \times 10^{6}\right.$ spores $\left./ \mathrm{mL}\right)$. Petri dishes with sprayed seeds were placed in high humidity and incubated at $25 \mathrm{C}$. Individual seeds were scored for A. flavus colonization, using a rating scale of 1 to 4 as described by Thakur et al. (2000).

\section{Results}

Pods from cross pollinations using $A$. chiquitana as the pollen parent appeared similar to $A$. hypogaea but were mostly single seeded. Thirtyfour percent of the pollinations formed pods from 

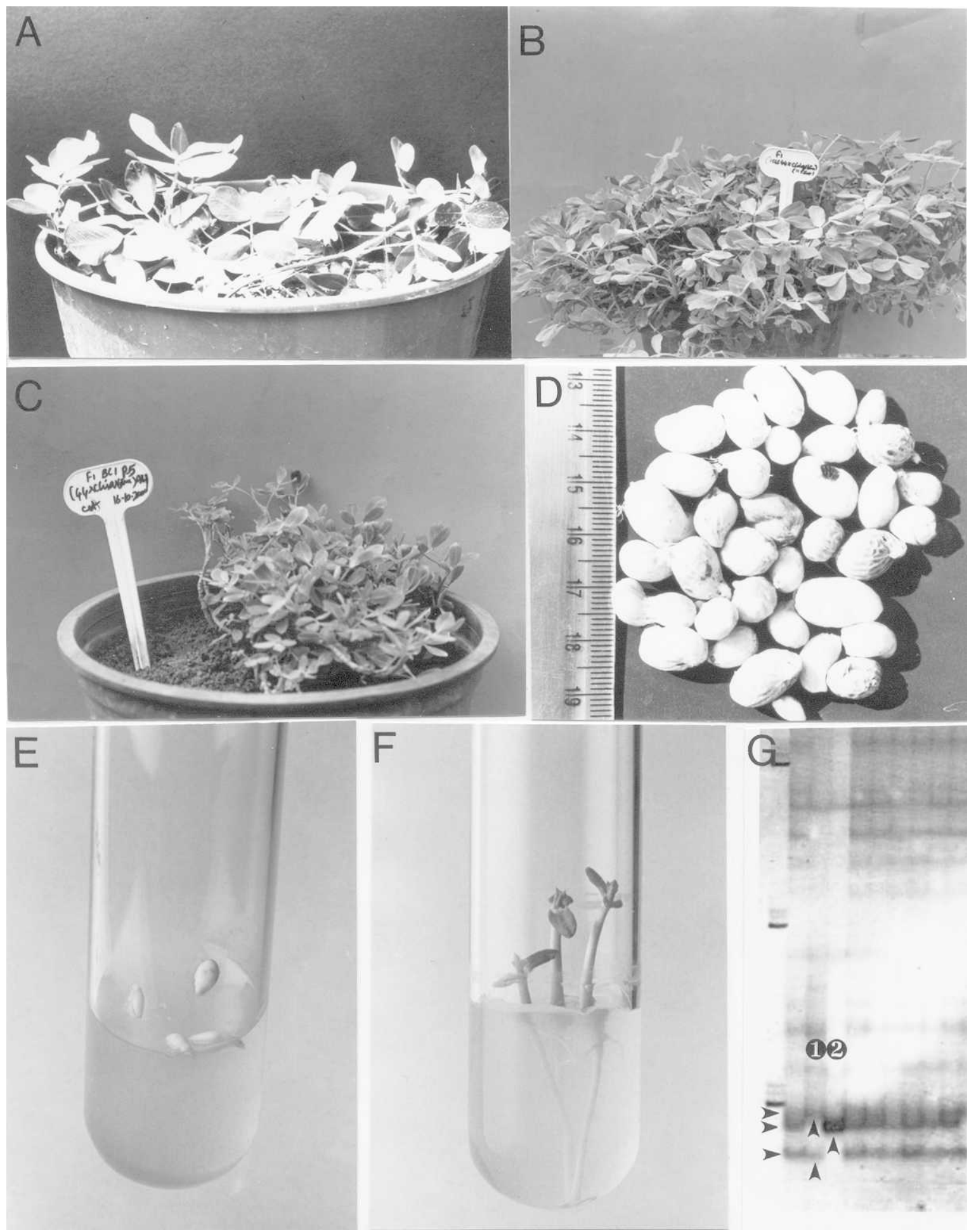

Fig. 1. Crossability between $A$. hypogaea and $A$. chiquitana.

A. Plant of the wild species $A$. chiquitana.

B. $F_{1}$ hybrid plant from the cross $A$. hypogaea $\times A$. chiquitana.

C. $\mathrm{BC}_{1}$ hybrid plant of the cross $A$. hypogaea $\times$ A. chiquitana.

D. $\mathrm{BC}_{2}$ pods showing mostly single seeded and a few double seeded pods.

E. Large but immature embryos from the cross $A$. hypogaea $\times$ A. chiquitana on germination medium.

F. Germinating seedlings from the cross $A$. hypogaea $\times$ A. chiquitana.

G. SSR-3F1, fragment length 290 base pairs: analysis of the parents and the hybrids. From left to right: $1^{\text {st }}$ lane: Molecular weight marker; $2^{\text {nd }}$ lane: $\mathrm{F}_{1}$ hybrid, hybrid bands have been marked with arrows; $3^{\text {rd }}$ lane: Arachis hypogaea, the female parent, marked no. $1 ; 4^{\text {th }}$ lane: $A$. chiquitana, the male parent, marked no. 2; Lanes 5 to 10: $F_{1}$ hybrids, hybrid bands have been marked with arrows. 
the cross $A$. hypogaea $\times A$. chiquitana. Aborted and dried seeds were obtained if harvested beyond $30 \mathrm{~d}$. Forty-seven percent of the ovules (immature seeds) were large $(>4.0 \times 2.5 \mathrm{~mm})$, rest of the seeds less than $4.0 \mathrm{~mm}$ long were discarded. A total of 25 large ovules were dissected and embryos were cultured (Fig. 1E). Approximately $30 \mathrm{~d}$ was required for the embryos to respond to culture and develop into healthy seedlings (Fig. 1F) or form multiple shoots. If the hybrid pods with large ovules were allowed to dry, then they did not germinate in vivo or in vitro. Eleven hybrid plants were obtained (Fig. 1B). One plant did not reach the flowering stage, in spite of having normal vegetative growth. The hybrid plants had intermediate morphology with the leaf shape resembling that of A. chiquitana. All the 10 plants flowered profusely and pollen fertility ranged from 5 to $24 \%$.

Hybrid plants were backcrossed using $A$. hypogaea as the pollen parent. It was possible to obtain mature $\mathrm{BC}_{1}$ pods from one hybrid plant, whereas the remaining plants did not set mature seeds. $\mathrm{BC}_{1}$ pods were mostly single seeded and abnormal in their shape. Twelve $\mathrm{BC}_{1}$ hybrid plants were obtained (Fig. 1C), from which two remained in the juvenile stage with little vegetative growth. Four of the plants had good vegetative growth but did not reach the flowering stage. Six hybrid plants flowered after propagation for at least $180 \mathrm{~d}$. Pollen fertility in the $\mathrm{BC}_{1}$ hybrids ranged from 18 to $24 \%$, and were tetraploids $(2 n=4 x=40)$ which was confirmed by pollen diameter analysis. Mature $\mathrm{BC}_{2}$ seeds were obtained, but in small number, ranging from one to 11 seeds. Most of the pods were single seeded, and the few double seeded pods produced severe constriction between the seeds (Fig. 1D).

Four SSR markers showed polymorphism between the two parents. SSR 3F1 (Fig. 1G) showed heterozygosity between the two parents. Two unique bands were consistently seen in $A$. hypogaea, whereas $A$. chiquitana had one distinct band. All seven $F_{1}$ hybrids used in the study showed the presence of bands from both the parents.

To check if it was possible to transfer resistance to $A$. flavus colonization, seeds were subjected to $A$. flavus colonization following the method described by Thakur et al. (2000). Large variation was observed with respect to fungal colonization. Individual seeds were scored for surface colonization, and the results showed six seeds which had the rating of 1 (resistant), and 12 seeds had the rating of 4 (susceptible). The other seeds showed a rating of 2 or 3 . Since the method to test for the presence of aflatoxin is destructive to the seed (Devi et al., 2000), seeds were not sacrificed to test for the presence of aflatoxin. Once large numbers of seeds are produced, the logical next step will be to test seeds for $A$. flavus colonization and aflatoxin production.

\section{Discussion}

Wild species from section Arachis have been used in the improvement of peanut because they are compatible with cultivated peanut (Mallikarjuna et al., 2004). Although there have been various attempts in the past to cross wild species from other sections of Arachis, the attempts have not been successful because of barriers to crossability. Even if the barriers are overcome by the use of various in vivo or in vitro techniques, many times the hybrid plants are male and female sterile or they do not mature into the reproductive phase (Stalker and Simpson, 1995). Tissue culture techniques first developed to recover aborting embryos from crosses between $A$. hypogaea and A. glabrata (Mallikarjuna and Sastri, 2002), a wild species belonging to section Rhizomatosae, can be applied to recover hybrid embryos from other intersectional crosses (Mallikarjuna, 2002) as is evident from the present study. Crosses between $A$. hypogaea and $A$. paraguariensis (ICG 8130; PI $337350 \mathrm{KCF} 11462$ ) and $A$. hypogaea and $A$. appressipila (ICG8945; PI 468149; GK 30003) were obtained by the use of tissue culture techniques. The hybrids had profuse vegetative growth but did not reach reproductive stage (Mallikarjuna, unpubl. data), and were considered genetic dead-ends. The hybrids obtained in the present study as well as with other A. hypogaea intersectional hybrids indicate that a generalized statement cannot be made that all hybrids involving wild species outside section Arachis are genetic dead-ends.

It would be of interest to pursue crosses involving $A$. chiquitana because it is one of the few wild species that can be crossed with $A$. hypogaea to potentially transfer resistance to $A$. flavus. The other species that have resistance to $A$. flavus are $A$. pusilla and $A$. triseminata ( Thakur et al., 2000), A. duranensis and $A$. cardenesaii (Xue et. al., 2004), the latter two species are cross compatible with $A$. hypogaea.

The present study shows that the crossability barriers are not rigid and with the manipulation of embryo rescue techniques available for peanut, many more species from different sections may be successfully crossed with cultivated peanut. 


\section{Literature Cited}

Ferguson, M.E., M. Burrow, S.R. Schulze, P.J. Bramel, A.H. Paterson, S. Kresovich, and S. Mitchell. 2004. Microsatellite identification and characterization in peanut (A. hypogaea L.). Theor. Appl. Genet. 108:1064-1070.

Ghewande, H.P., G. Nagaraj, and P.S. Reddy. 1989. Aflatoxin research at the National Research Center for Groundnut, pp. 237-243. In D. McDonald and V.K. Mehan (eds.). Aflatoxin Contamination of Groundnut: Proc. Intl. Workshop, 6-9 Oct. 1987, ICRISAT, Patancheru, A.P., India.

Gong, Y.Y., K. Cardwell, A. Hounsa, S. Egal, P.C. Turner, A.J. Hall, and C.P. Wild. 2002. Dietary aflatoxin exposure and impared growth in young children from Benin and Togo: Cross sectional study. British Medical J. 325:20-21.

Gregory, M.P., and W.C. Gregory. 1979. Exotic germplasm of Arachis L. interspecific hybrids. J. Hered. 70:185-193.

Househam, K.C., and H.K. Hunt. 1991. Aflatoxin exposure and its relationship to kwashiorkor in African children. J. Tropical Pediatrics, 37:300-302.

Krapovikas, A., and W.C. Gregory. 1994. Taxonomia del genero Arachis (Leguminosae). Bonplandia 8:1-186.

Mallikarjuna, N. 2002. Gene introgression from A. glabrata into $A$. hypogaea, A. duranensis and A. diogoi. Euphytica 124:99-105.

Mallikarjuna, N., and D.C. Sastri. 2002. Morphological, cytological and disease resistance studies of the intersectional hybrids between Arachis hypogaea and A. glabrata. Euphytica 126:161167.

Mallikarjuna, N., Suresh Pande, D.R. Jadhav, D.C. Sastri, and J.N. Rao. 2004. Introgression of disease resistance genes from Arachis kempff-mercadoi into cultivated groundnut. Plant Breeding 123:573-576.
Murashige, T., and F. Skoog. 1962. A revised medium for rapid growth and bioassays with tobacco tissue cultures. Physiol Plant 15:473-497.

Pande, S., and J.N. Rao. 2001. Resistance of wild Arachis species to late leaf spot and rust in greenhouse trials. Plant Disease. $85: 851-855$

Saghai-Maroof, M.A., K.M. Solinam, R.A. Jorgonsan, and R.W. Allard. 1984. Ribosomal DNA spacer length polymorphism in barley. Mendelian inheritance, chromosomal location and population dynamics. Proc. Natl. Acad. Sci., (USA) 81:8014-8018.

Stalker, H.T. 1981. Hybrids in the genus Arachis between sections Erectoides and Arachis. Crop Sci. 21:359-362.

Stalker, H.T., and C.E. Simpson. 1995. Genetic Resources in Arachis pp. 14-53. In H.E. Pattee and H.T. Stalker (eds.). Advances in Peanut Science. Amer. Peanut Res. Educ. Soc., Stillwater, OK.

Thakur, R.P., V.P. Rao, S.V. Reddy, and M. Ferguson. 2000 Evaluation of wild Arachis germplasm accessions for in vitro seed colonization and aflatoxin production by Aspergillus flavus. Int Arachis Newsletter 20:44-46.

Thirumala Devi, K., M.A. Mayo, G. Reddy, S.V. Reddy, P. Delfosse, and D.V.R. Reddy. 2000. Production of polyclonal antibodies against ochratoxin A and its detection in chilies by ELISA. J. of Agric. and Food Chem. 48:5079-5082.

Waliyar, F., A. Ba, H. Hassan, S. Bonkoungou, and J.P. Bose. 1994 Sources of resistance to Aspergillus flavus and aflatoxin contamination in groundnut in West Africa. Plant Disease 78:704-708.

Williams, J.G.K., A.R. Livak, J.A. Rafalski, and S.V. Tingey. 1990 DNA polymorphisms amplified by arbitary primers are useful as genetic markers. Nucleic Acids Res. 18:6531-6535.

Xue, H.Q. T.G. Isleib, H.T. Stalker, G.A. Payne, and G. Obrian 2005. Evaluation of Arachis species and Interspecific Tetraploid Lines for Resistance to Aflatoxin Production by Aspergillus flavus. Peanut Sci. 31:134-141. 